Article

\title{
Topological Transitions in Collective Housing Units of South Korea
}

\author{
Sungil Ham and Hyunsoo Lee * \\ Department of Interior Architecture and Built Environment, Yonsei University, Seoul 03722, Korea; \\ archispace@yonsei.ac.kr \\ * Correspondence: hyunsl@yonsei.ac.kr; Tel.: +82-2-2123-3136
}

Academic Editor: Marc A. Rosen

Received: 20 October 2016; Accepted: 23 December 2016; Published: 28 December 2016

\begin{abstract}
Collective housing in South Korea started its introduction from the early 1960s, and became the most important residential type in 50 years. Because of the importance of collective housing in many countries and in South Korea, many studies on collective housing have been conducted. Although these studies can provide criteria and methods quantitatively and objectively, classification of types is dependent on researcher's judgment, and classification of type without considering the plan's time of creation has a limitation to derive changes in type patterns. The purpose of this study is to analyze changes quantitatively and objectively in patterns of spatial structure and to derive types of spatial structure of collective housing in South Korea algorithmically using a time-based joining method that produced a phylogenetic tree using similarities of spatial structure and plan creation time. This study also supports a research frame for researchers who want the use of a time-based joining method. The study analyzed the relationships of changes in spatial structures of 890 collective housing unit plans in Seoul, South Korea, constructed from 1970 to 2015.
\end{abstract}

Keywords: collective housing; time-based joining method; plan type; phylogenetic tree

\section{Introduction}

In South Korea in 2010, collective housing accounted for 59\% of all residences [1]. Collective housing complexes were introduced to South Korea beginning in the early 1960s, and within 50 years, they had become the most common type of residence. Due to the importance of collective housing in South Korea, many studies have been conducted on this subject. In particular, since the spatial configuration of residential units has significant meaning in terms of the social and cultural context, a number of studies on spatial topology have been conducted [2,3]. Quantitative studies [4-6] have been conducted on the classification of spatial structures using the analysis values of space syntax [7], or on the classification of plans by decomposing spatial structures into segment units. Although these studies can provide quantitative and objective criteria and methods, the classification of residence types is dependent on the researcher's judgment; if the time at which the plans were created is not considered, there is a limitation in determining the changes in the patterns of residence types.

The present study aims to analyze changes-both quantitatively and objectively-in the pattern of spatial structures, and to determine the topological transitions in units of collective housing called apartments whether they are rented or owned by the tenants in South Korea via the time-based joining method (TBJ) $[8,9]$, which produces a phylogenetic tree using the similarities in spatial structures and the time at which the plan was created. This study also supports a research frame for researchers who want the use of a time-based joining method and to look at sustainability from the perspective of planning through a transition in spatial structure of collective housing units. Spatial structure is also changed according to the social, economic, and cultural factors of the times. Specific plans gradually 
change, maintaining sustainability, but the cases which are not preferred disappear. The practices of sustainable spatial structure are derived through this study.

The present study is a follow-up to a previous study (three-bay, two-unit-staircase-type) [10], and it targets the most typical type of apartments before three-bay type; i.e., two-bay, two-unit-staircase-type (as opposed to multiple-unit-corridor type) collective housing plans in Seoul, with a usable floor area of $85 \mathrm{~m}^{2}$. A two-bay unit is a unit whose main area is composed of two bays, typically a living room and a main room. A two-unit-staircase-type plan is a type of apartment floor plan in which two units on the same floor share a staircase, and often also an elevator shaft.

In this study, 980 unit plans of apartments that were built in Seoul from 1979 to 2015 are analyzed. The analysis method is as follows: the TBJ methodology is used to create phylogenetic trees, and then family classification and changes in types over time are analyzed based on the system map.

The present paper is organized as follows: In Section 2, the premises that enable architectural planning and design studies through phylogenetic trees are explained. Section 3 gives overviews of the literature, along with the methodologies to evaluate the similarities of spatial structures, the methodologies to prepare time-based phylogenetic trees, and the characteristics of the apartments and dwellings in South Korea. In Section 4, the phylogenetic tree is created. Each family is listed to analyze the changes in patterns. This section also analyzes the spatial structure and change of the three main areas based on the phylogenetic tree. In Section 5, the contributions and limitations of the study are provided.

\section{Premises of Phylogenetic Trees in Architectural Planning and Design Studies}

The present study is based on the premise that buildings-particularly houses and apartments-have consistency in terms of certain factors given by customs and traditions, yet continuously change according to alterations in social systems, regulations, and life patterns. Although the principles of spatial organization in residential spaces change, the revised principles are often similar to the previously existing ones.

Bafna $[11,12]$ defined the sociological principles and structures of spaces that cannot be arbitrarily changed by architects as genotypes. Such principles are defined as codes that determine the phenotype of a certain class of individual forms and patterns [13]. The present study addresses the principle of spatial organization, and defines genotypes as combinations of rooms that indicate the typical characteristics of spaces.

Similarities and differences in spatial organization allow families for architectural spaces to evolve and develop. The term family originates in biology, where it refers to the history of evolution or the transmission patterns of biota. In this study, a family is a certain group of architectural plan types that share similar characteristics [14]. A family is generated by finding the most similar plan types and connecting them to each other [8].

Phylogenetic trees have been used as a foundation for the classification of living things. The humanities, sociology, and computer engineering fields have used phylogenetic trees to explain processes and to establish various hypotheses. Phylogenetic studies have been criticized extensively because of their excessive biological analogies and applications. However, they have contributed to the architectural field by providing a new framework for research. The present study analyzes the meaning behind changes in the spatial organization of apartments by determining their similarities through phylogenetic trees prepared by the time-based joining method.

\section{Overview}

In this section, methodologies to analyze the similarities between graphs that compare spatial structures are used, and different preparation methodologies for phylogenetic trees are considered, to determine the processes of changes to apartments in South Korea, from their introduction until now. 


\subsection{Graph-Based Spatial Structure Similarity Analysis}

In architecture, spatial connections are expressed using node-edge graph. In a graph, a room (object) is the minimum unit that constitutes a building. Studies that analyze the similarities between spatial structures by calculating the costs of processes to make two graphs match have been conducted. The studies calculating the similarities between graphs have also been conducted in diverse areas and across methods; not only in architecture, but also in structural pattern recognition, computer vision, and database schema comparisons.

Samfeliu and $\mathrm{Fu}$ [15] divided the methodologies for measuring the similarities between graphs into two categories: feature-based distances and cost-based distances. Using feature-based distances, the characteristics to be compared are extracted from graphs; thus, two points can be compared by specifying distances. With cost-based distances, the distance between two graphs is measured by calculating the minimum cost of the editing operations (including deletions, insertions, and substitutions of nodes and edges) required to transform the first graph into the second graph [16].

In addition to these two methodologies, graph matching and subgraph isomorphism have been used to compare graphs. Recently, a methodology that analyzes structural similarity has been constructed in which similarity scores are calculated based on the premise that graphs should be similar when the nodes adjacent to the edges of two graphs are also similar. In this methodology, the scores of the similarities between individual elements are transferred depending on the adjacent element in each stage. This iterative methodology is affected by the HITS (Hyperlink-Induced Topic Search) algorithm [17] introduced by Kleinberg. Algorithms that use the methodology include the similarity-flooding algorithm [18] proposed by Melnik, the SimRank algorithm [19] proposed by Jeh and Widom, the scoring method for phylogenetic tree construction [20] proposed by Heymans and Singh, and the vertex similarity method [21] proposed by Leicht.

Iterative methodologies are constructed between graphs in three steps. In the first stage, the actual relations of the subjects are formed into graphs consisting of nodes and edges. In the second stage, one pair of relations between the connected nodes is compared with one pair of node-node relations from another graph (i.e., a pairwise comparison). Through this comparison, a distance matrix is formed between the two compared node pairs. Using this matrix, the optimum similarity is obtained through a Hungarian algorithm analysis. The second process is implemented through graph matching. Among the many graph-matching algorithms explained earlier, in the present study, the bipartite methodology [20] proposed by Heymans and Singh was used. In the study conducted by Heymans and Singh, the similarity between certain species was evaluated through the similarity levels of metabolic pathway graphs. The results were constructed into phylogenetic trees, and the phylogenetic trees were compared with existing biological phylogenetic trees. This is in line with the aim of the current study, which constructed phylogenetic trees to determine the similarities between apartments. In Section 3, the preparation of the phylogenetic trees is shown, and the similarities between the spatial structures of apartments is analyzed.

\subsection{Time-Based Joining Method for Generating Phylogenetic Trees}

A TBJ method is employed to create a phylogenetic tree that considers the time in which a plan was generated in order to investigate the families and changes in the spatial structures of apartments. The TBJ method assumes that dwelling places—-particularly apartments-remain consistent as a result of factors such as traditions and customs (which are not easily changed), while changes result from factors such as the social system, regulations, and living patterns. The similarities and changes in spatial configurations have evolved, forming a phylogeny between architectural spaces; a genotype configures a space, while a new design phenotype is generated based on this genotype.

The method for generating a phylogenetic tree using the TBJ method is as follows: for the target plans in this study, whether a specific plan type (classification group) was preceded by other plan types (classification group) can be determined based on the building date. A plan type that chronologically precedes other plan types is a precedent candidate; if similarities are found between the spatial 
structures in two plans and one of the plans is created earlier than the other, then the earlier plan is the precedent plan type. A phylogenetic tree can be produced by connecting all the plan types that have such precedent-descendent relationships. The generation method is shown in Figure 1 and explained below:

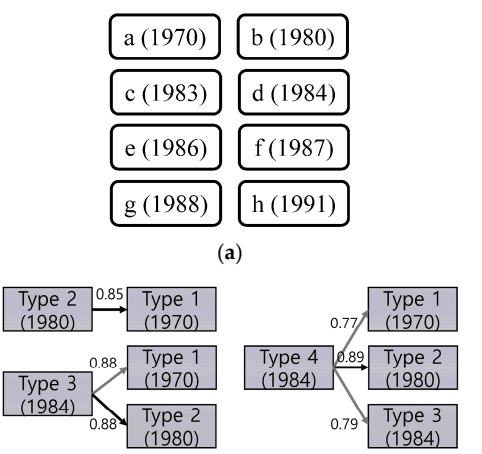

(c)

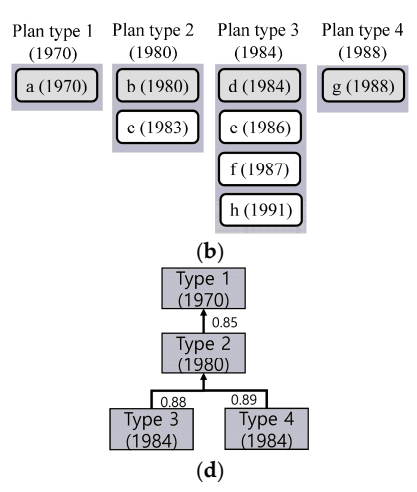

Figure 1. Generation process for the phylogenetic tree.

(a) There are eight cases (a to $\mathrm{h}$ plans) as analysis targets.

(b) A set of plans that have identical spatial structures is defined as a plan type, and these are collected as a group. The generation time for each plan type is determined based on the earliest completion date.

(c) The similarities of each plan type with the plan types that are chronologically earlier are calculated, and the plans with the highest similarity are paired. In this pair, the plan type that is chronologically earlier becomes the precedent, while the plan type that is chronologically later becomes the descendent.

(d) The above procedure is iterated to derive the precedent-descendent relationships, which are then represented as a single connected relationship to create a phylogenetic tree. In the present study, the similarity between spatial structures was calculated using the bipartite methodology proposed by Heymans and Singh [20].

\subsection{Characteristics of Dwellings in South Korea}

In the spatial organization of traditional architecture established before 1920, the floor was created first at the center of a house (as shown in Figure 2), and rooms were arranged around the floor. In addition, narrow wooden porches running along the outside of rooms provided access to individual rooms. The kitchen was directly connected to the outside of the building and was connected to the main room or to an auxiliary space for the preparation of meals. The bathroom was arranged outside of the building for hygienic reasons. In detached houses, immediately before the introduction of apartments, narrow wooden porches running along the outside of rooms disappeared, and the floor became a central space that mediated individual spaces. In addition, the bathroom was brought indoors.

A characteristic of South Korean dwellings is floor heating. Sitting culture was dominant for some time, even after the introduction of apartments. No furniture such as dining tables and beds existed, and each room was a multipurpose space in which eating, sleeping, and leisure activities were all performed. Another characteristic South Korean dwellings stems from the climate of South Korea. Due to hot and humid summers and cold winters, houses are currently designed to face South to account for sunshine and ventilation. Therefore, bedrooms and the living room are arranged to face South, and other spaces, such as the kitchen, dining room, and bathroom, are arranged to face North. Consequently, spatial arrangements are restricted. These characteristics of South Korean dwellings are taken into account in the present study to examine the meanings of changes in the spatial organization of apartments. 


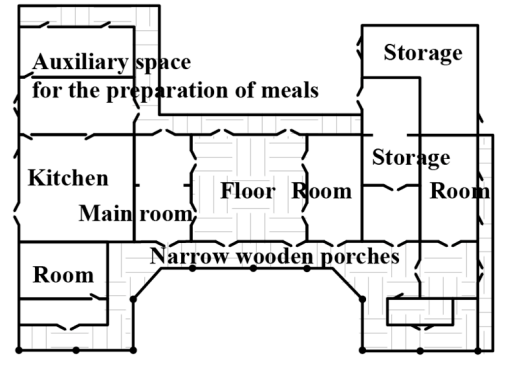

(a)

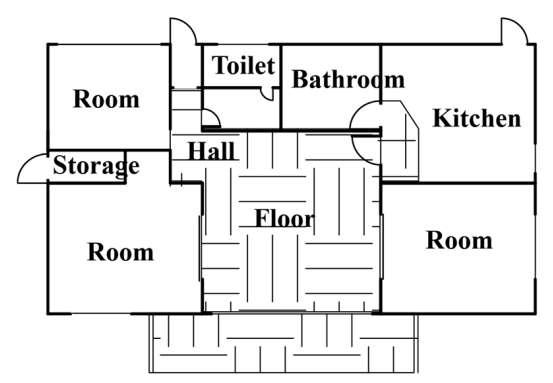

(b)

Figure 2. Examples of a traditional Korean-style house and detached house. (a) Traditional Korean-style house (Head house of Sun family: 1921); (b) Detached house (Reconstruction of house by Korea Housing Corporation: 1959).

\subsection{Introduction and Changes in Apartment Structures}

In the 1960s and 1970s, industrialization occurred rapidly as a result of state-led economic development. Due to this rapid economic growth, population growth was concentrated in urban cities, and housing shortages accelerated due to the explosive increase in demand for land and housing. To overcome this problem, apartment houses emerged as one of the solutions. Starting with apartments in Mapo in 1962, a large number of apartments were built, and these became a typical type of urban residence in South Korea. In the early days of apartment construction, experimental apartments were built, such as the Susek and Jangchung apartments, and several standard apartment plans gradually emerged. This emergence was due not only to the complex socioeconomic and legal factors, but also to the specific residential preferences of housing consumers, which led to large-scale construction.

The introduction of apartments and the process and characteristics of change in apartment types are presented below.

In the 1960s, as a result of economic development and industrialization, rural exodus increased alongside an overall population increase, and this led to rapid urbanization and rapidly degraded the quality of residential environment. The Mapo apartments (1962) was the first of the apartment-oriented residential complexes.

The characteristics of housing construction in the 1970s can be divided into the development of complexes by public institutions, such as the KHC (Korea Housing Corporation), and large-scale apartment construction by private companies. This had a number of side effects, including excessive standardization of apartment structures by adopting Western planning principles based on the functionality of unit housing, such as providing a continuous array of the same apartment type and the same unit plans [22].

As of the 1970s, planning principles for apartment construction were routinely maintained, and apartments in the 1980s consolidated the backwardness of excessive standardization for apartment structures and planning. Furthermore, most residential complexes became high-rises with high-density due to the increase in land price.

Skyrocketing price increases for land and housing emerged at the end of the 1980s due to the unprecedented economic boom. The number of new housing constructions increased exponentially, as did the size of the housing market. Since 1987, the development of individual complexes by private construction companies in housing land-development districts has become more common [23].

The demand for apartments decreased due to the economic crisis in 1997. As a result, the existing supplier-led housing market shifted to a consumer-based market. Accordingly, product planning, brand development, advertisement and marketing, identification of a consumer's preference, and the diversification of house plans were utilized to meet the requirements of consumers. This trend accelerated following the deregulation of sale prices for houses in 1998.

Although the regulation of sale prices was temporarily re-enforced in 2005 due to a sudden increase in apartment prices, the regulation of these sale prices was abolished again in September 2012 
due to a recession in the housing sector. The Enforcement Ordinance of the Building Act allowed changes in the structure of balconies in December 2005, which put an end to the controversial balcony-expansion debate. Though it was illegal, changing the structure of balconies had been done customarily. By including balcony space as indoor residential space, apartment plans were developed to utilize the extended balcony space, which resulted in different plans in terms of the ratio between living rooms and bedroom [24].

In Section 4, the research methodology and background presented in this section are used to create and analyze a phylogenetic tree using the similarity of the spatial structure of apartment units.

\section{Generation of the Phylogenetic Tree}

In this section, a phylogenetic tree is generated using the TBJ method to determine the changes in the spatial structures of apartments. A phylogenetic tree is generated using the similarities between spatial structures, as these represent connection relations between spaces. The changes in spatial structures and characteristics between the different types of apartments are analyzed using the generated phylogenetic tree. The target apartment has an area of $85 \mathrm{~m}^{2}$, which is the most common size in South Korea. The present study analyzes 890 apartments with a two-bay staircase unit plan that were built in Seoul between 1970 and 2015, as this was the most popular type before the three-bay unit plan became popular.

\subsection{Generation of a Phylogenetic Tree for Spatial Structures}

As explained earlier, the present study intended to analyze the types of changes in the spatial structures of apartment unit plans; that is, the connection relations between their rooms. Therefore, the relations between the rooms in the unit plans were expressed using j-graphs. The j-graphs contain only information on the relations between rooms (depth) that can be accessed from the entrance. That is, information on the sizes and locations of the rooms was excluded from the present study. In addition, this study does not include spaces that cannot be considered as independent rooms, such as utility rooms, built-in closets, balcony, and storage.

In this study, $890 \mathrm{CAD}$ drawings were made as input data for the generation of a phylogenetic tree. Each room and door was made up of polylines. Using the intersection of these polylines, j-graphs were extracted. Figure 3 shows the j-graphs derived from eight plans.

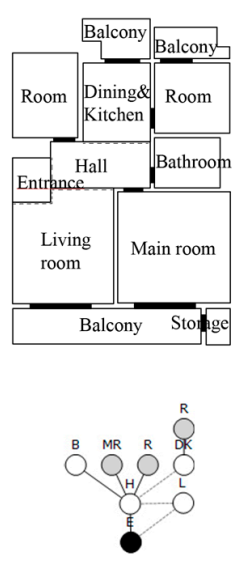

Sillim Hyundai

(1987)
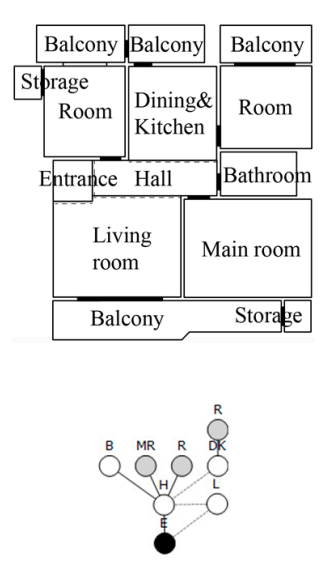

Ssangmoon Dongik

(1991)
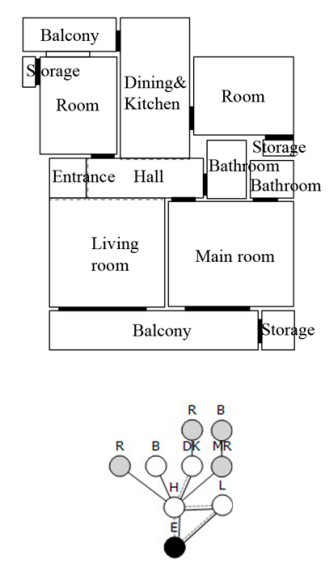

Banghak Byucksan

(1988)
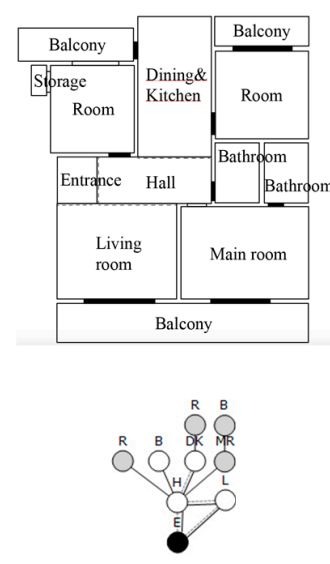

Ssangmoon Kumho

(1989)

Figure 3. Example architectural plans of collective housing units and j-graphs for the generation of a phylogenetic tree (R: Room, MR: Main room, L: Living room, H: Hall, E: Entrance, D: Dining, K: Kitchen, B: Bathroom, P: Powder room or dressing room, the number between parentheses: move-in year). 
Eight hundred and ninety j-graphs were grouped into 58 planar shapes with the same homomorphism. Figure 4 shows the four previously-created j-graphs grouped into two plan types with the same homomorphism. Sillim Hyundai (1987) and Ssangmoon Dongik (1991) are grouped into Plan Type 9 (1987), because their j-graphs are identical.

\section{Plan type 9 (1987)}

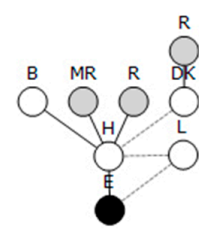

Sillim Hyundai (1987)

Ssangmoon Dongik (1991)

\section{Plan type 11 (1988)}

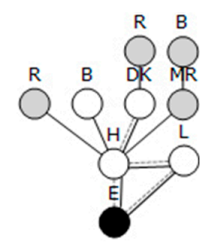

Banghak Byucksan(1988)

(Ssangmoon Kumho (1989)

Figure 4. Plan type groups with the same spatial structure, and j-graphs that represent them.

The 890 prepared j-graphs were grouped according to topology into 58 groups, as shown in Table 1. The ID column of Table 1 refers to the 58 plan types, and the j-graphs of the plan types of the individual IDs were mapped using the IDs in Figure A1 in the Appendix A. In Table 1, the year (count) column refers to the years and count of construction. The count column refers to the individual cases of the plans (890 in total). The matching ID column shows the plan types with the earliest years of construction and the highest similarity, which was determined by comparing the similarity scores of the 58 plan types (i.e., the TBJ methodology). The similarity score column shows the similarity scores to the matched plan types. The prepared phylogenetic trees are shown in Figure 2.

The Family and Spatial Genotype column is a family in which each plan type belongs, and genotypes that represent the combination of rooms. Genotypes are shown in Figure 9. In this study, 58 plan types are phenotypes of these genotypes, and the families are formed through a common genotype.

Table 1. The 58 plan types of two-bay staircase apartments between 1979 and 2015.

\begin{tabular}{|c|c|c|c|c|c|}
\hline ID & Year (Count) & Count & Similarity Score & Matching ID & $\begin{array}{l}\text { Family and Spatial } \\
\text { Genotype }\end{array}$ \\
\hline 1 & $79(1)$ & 1 & 0 & 0 & 2A, KB-HA-EA-MA \\
\hline 2 & $\begin{array}{l}80(1), 82(1), 83(9), 84(4), 85(5), 86(6), 87(3) \\
88(8), 89(1), 90(2), 93(1), 94(1)\end{array}$ & 42 & 0.817 & 1 & 2B, KA-HA-EA-MA \\
\hline 3 & $82(1), 84(1), 88(2), 89$ (1), 91 (1), 92 (1) & 7 & 0.857 & 1 & 2A, KB-HA-EA-MA \\
\hline 4 & $\begin{array}{l}82(1), 83(1), 86(1), 88(1), 92(1), 93(1), 94(1) \\
98(1), 01(1), 04(2), 05(1), 06(1), 07(1)\end{array}$ & 14 & 0.881 & 2 & 2B, KA-HA-EA-MA \\
\hline 5 & $84(1)$ & 1 & 0.687 & 2 & 2B, KA-HA-EA-MA \\
\hline 6 & $\begin{array}{l}86(1), 88(6), 89(9), 90(4), 91(12), 92(18), \\
93(12), 94(5), 95(18), 96(11), 97(5), 98(6), \\
99(11), 00(3), 01(7), 02(5), 03(1), 04(2), \\
05(2), 06(1), 07(1), 09(1), 13(1)\end{array}$ & 141 & 0.886 & 3 & 2A, KB-HA-EA-MB \\
\hline 7 & $86(1)$ & 1 & 0.583 & 1 & 2A, KD-HA-EB-MA \\
\hline 8 & $87(1)$ & 1 & 0.736 & 7 & 2A, KD-HA-EA-MA \\
\hline 9 & $87(1), 91(1)$ & 2 & 0.886 & 3 & 2A, KA-HA-EB-MA \\
\hline 10 & $87(1)$ & 1 & 0.818 & 6 & 2A-2, KB-HA-EB-MB \\
\hline 11 & $\begin{array}{l}88(2), 89(1), 90(5), 91(9), 92(15), 93(16), \\
94(26), 95(15), 96(19), 97(37), 98(52), 99(69), \\
00(52), 01(28), 02(24), 03(25), 04(15), 05(7), \\
06(6), 07(4), 14(1), 15(1)\end{array}$ & 429 & 0.908 & 10 & 2A-2, KB-HA-EB-MB \\
\hline 12 & $88(1), 92(1), 95(2), 97(1), 99(1), 08(1)$ & 7 & 0.874 & 6 & 2A-1, KC-HA-EA-MB \\
\hline
\end{tabular}


Table 1. Cont.

\begin{tabular}{|c|c|c|c|c|c|}
\hline ID & Year (Count) & Count & Similarity Score & Matching ID & $\begin{array}{l}\text { Family and Spatial } \\
\text { Genotype }\end{array}$ \\
\hline 13 & $88(1)$ & 1 & 0.888 & 2 & 2A-1, KA-HA-EA-MA \\
\hline 14 & $\begin{array}{l}89(3), 90(2), 92(1), 93(3), 94(6), 95(6), 96(1) \\
97(1), 00(7), 01(4), 02(2), 04(1), 06(1)\end{array}$ & 38 & 0.875 & 12 & 2A-1, KC-HA-EA-MB \\
\hline 15 & $\begin{array}{l}89(1), 90(1), 91(4), 92(7), 93(5), 94(8), 95(1) \\
96(5), 97(8), 98(13), 99(10), 00(9), 01(6), \\
02(2), 03(7), 04(4), 05(5)\end{array}$ & 96 & 0.891 & 11 & 2A-2, KC-HA-EB-MB \\
\hline 16 & $90(1)$ & 1 & 0.875 & 12 & 2A-1, KC-HA-EA-MB \\
\hline 17 & $90(1)$ & 1 & 0.889 & 11 & 2A-2, KB-HA-EB-MB \\
\hline 18 & $90(1)$ & 1 & 0.721 & 15 & 2A-2, KD-HA-EB-MB \\
\hline 19 & $91(1)$ & 1 & 0.881 & 2 & 2B, KA-HA-EB-MA \\
\hline 20 & $91(1), 92(1), 02(1), 05(1)$ & 4 & 0.901 & 6 & 2A-2, KB-HA-EA-MB \\
\hline 21 & $92(1), 93(1), 96(2), 98(1), 99(1)$ & 6 & 0.87 & 4 & 2B-3, KA-HB-EA-MB \\
\hline 22 & $92(2), 98$ (1), 99 (1), 00 (1), 04 (3), 05 (1), 07 (1) & 10 & 0.896 & 4 & 2B-2, KA-HA-EB-MB \\
\hline 23 & $92(1)$ & 1 & 0.8 & 17 & 2A-2, KA-HA-EB-MB \\
\hline 24 & $92(1)$ & 1 & 0.901 & 14 & 2A-1, KC-HA-EC-MB \\
\hline 25 & $92(1), 94(1), 95(1)$ & 3 & 0.827 & 11 & 2A-2, KC-HA-EC-MB \\
\hline 26 & $92(1)$ & 1 & 0.896 & 4 & 2B-4, KA-HA-EA-MB \\
\hline 27 & $93(1)$ & 1 & 0.827 & 18 & 2A-2, KE-HA-EB-MB \\
\hline 28 & $93(1)$ & 1 & 0.875 & 12 & 2A-1, KC-HA-EB-MA \\
\hline 29 & $93(1)$ & 1 & 0.878 & 14 & 2A-1, KD-HA-EA-MB \\
\hline 30 & $93(1), 96(1), 00(1)$ & 3 & 0.878 & 12 & 2A-1, KD-HA-EA-MB \\
\hline 31 & 94 (1), 95 (1), 97 (2), 99 (1), 00 (1), 02 (2) & 8 & 0.899 & 12 & 2A-1, KC-HA-EB-MB \\
\hline 32 & $94(1)$ & 1 & 0.899 & 27 & 2A-2, KD-HA-EA-MB \\
\hline 33 & $\begin{array}{l}94(2), 95(1), 97(1), 98(2), 99(1), 00(1), 01(2), \\
02(1), 03(2)\end{array}$ & 13 & 0.899 & 30 & 2A-1, KD-HA-EB-MB \\
\hline 34 & $94(1)$ & 1 & 0.853 & 30 & 2A-1, KD-HB-EA-MB \\
\hline 35 & $96(1)$ & 1 & 0.887 & 14 & 2A-1, KC-HA-EA-MA \\
\hline 36 & $97(1), 01(1)$ & 2 & 0.899 & 7 & 2A, KD-HA-EB-MB \\
\hline 37 & $98(1)$ & 1 & 0.878 & 7 & 2A, KC-HA-EB-MA \\
\hline 38 & $98(1)$ & 1 & 0.886 & 4 & 2B-1, KA-HA-EA-MB \\
\hline 39 & $99(1), 06(1)$ & 2 & 0.761 & 11 & 2A-2, KB-HA-EA-MB \\
\hline 40 & $99(1)$ & 1 & 0.887 & 16 & 2A-1, KC-HA-EA-MA \\
\hline 41 & $99(1)$ & 1 & 0.884 & 22 & 2B-2, KA-HA-EA-MB \\
\hline 42 & $01(1), 02(2), 03(1), 04(4), 07(1)$ & 9 & 0.9 & 10 & 2A-2, KB-HA-EB-MC \\
\hline 43 & $01(1), 02(1), 03(3), 04(1), 05(1), 06(3)$ & 10 & 0.95 & 10 & 2A-1, KB-HA-EB-MD \\
\hline 44 & $02(2), 03(3), 04(2), 05(1), 06(1)$ & 9 & 0.904 & 42 & 2A-2, KC-HA-EB-MC \\
\hline 45 & $02(1)$ & 1 & 0.853 & 38 & 2B-1, KA-HA-EA-MB \\
\hline 46 & $03(2)$ & 2 & 0.899 & 16 & 2A-1, KE-HA-EB-MB \\
\hline 47 & $03(1)$ & 1 & 0.746 & 25 & 2A-2, KC-HB-EA-MB \\
\hline 48 & $03(1)$ & 1 & 0.844 & 30 & 2A-1, KD-HA-EA-MC \\
\hline 49 & $03(1), 04(1)$ & 2 & 0.95 & 44 & 2A-2, KC-HA-EB-MD \\
\hline 50 & $04(1)$ & 1 & 0.8 & 47 & 2A-2, KB-HB-EA-MC \\
\hline 51 & $04(1)$ & 1 & 0.774 & 21 & 2B-3, KA-HA-EA-MC \\
\hline 52 & $04(1)$ & 1 & 0.909 & 44 & 2A-1, KC-HA-EA-MC \\
\hline 53 & $05(1)$ & 1 & 0.889 & 26 & 2B-4, KA-HA-EA-MC \\
\hline 54 & $05(1)$ & 1 & 0.836 & 39 & 2A-1, KB-HA-EA-MC \\
\hline 55 & $05(1)$ & 1 & 0.766 & 43 & 2A-1, KC-HB-EA-MC \\
\hline 56 & $05(1), 10(1)$ & 2 & 0.871 & 39 & 2A-2, KB-HA-EA-MB \\
\hline 57 & $06(1)$ & 1 & 0.892 & 23 & 2A-2, KA-HA-EA-MC \\
\hline 58 & $06(1), 11(1)$ & 2 & 0.909 & 48 & 2A-1, KD-HA-EB-MC \\
\hline
\end{tabular}


As shown in the phylogenetic tree in Figure 5, 58 plan types were branched into two families:

(1) A family in which the bedrooms were connected through the kitchen and dining room (DK) (Family 2A, 1979, Sungbo Apt. in Yeoksam-dong in Figure 6a).

(2) A family in which all spaces were connected through the hall (Family 2B, 1980, Samick Green Apt. 1 to 6-Cha B type in Figure 6b).

These were branched as shown in Figure 5. Family 2A was further branched as follows:

(1) A family in which the entrance and hall are connected (2A-1, plan type 12, 1988).

(2) A family in which the entrance, hall, and living room are interconnected (2A-2, plan type 10, 1987).

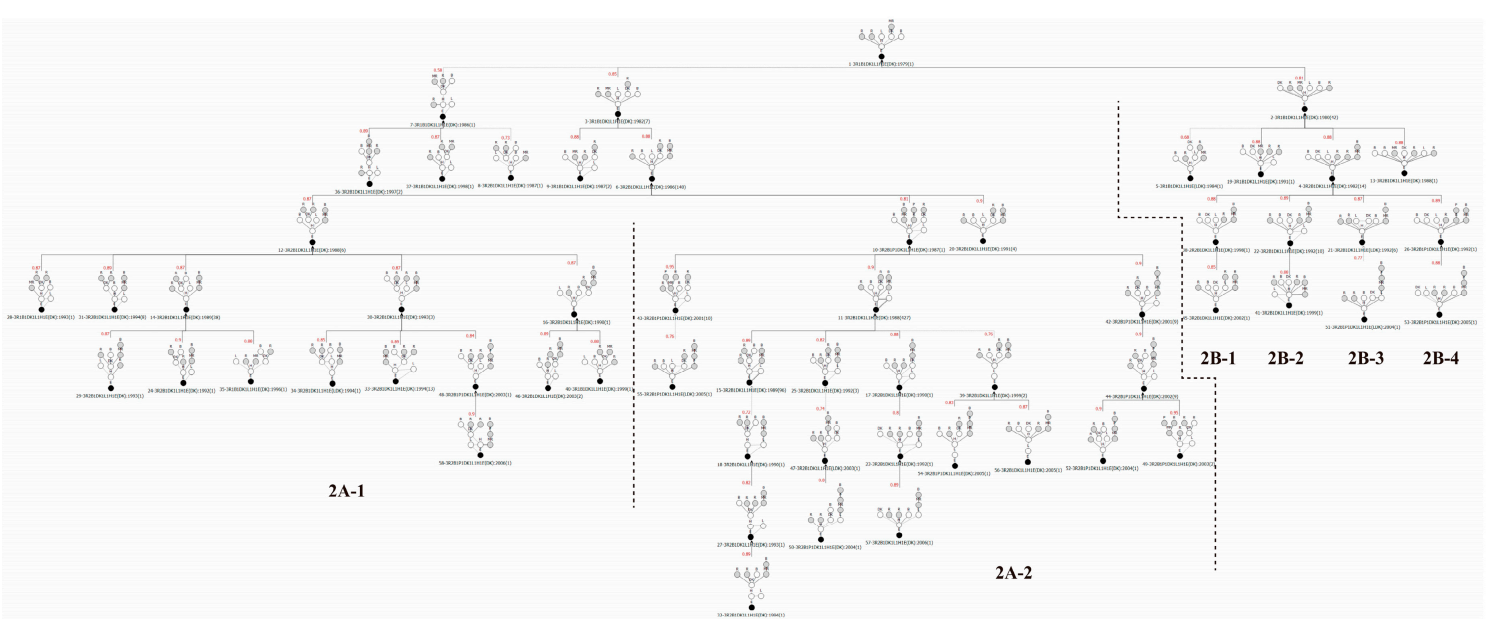

Figure 5. A phylogenetic tree for plan types of two-bay staircase apartments between 1979 and 2015.

Table 2 summarizes the features of families by group based on the phylogenetic tree.

Table 2. Features of families.

\begin{tabular}{llll}
\hline \multirow{3}{*}{ Family 2A } & $\begin{array}{l}\text { Family in which any spaces (rooms) } \\
\text { are connected through the kitchen } \\
\text { and dining room (DK) (DK-*) }\end{array}$ & 2A-1 & Only entrance is connected to the hall (E-H) \\
\cline { 3 - 4 } & 2A-2 & $\begin{array}{l}\text { Entrance, hall, and living room are } \\
\text { inter-accessible (E-H, E-L, L-H) }\end{array}$ \\
\cline { 3 - 4 } Family 2B & 2B-1 & Bedrooms are accessible via the living room (L-R) \\
\cline { 3 - 4 } $\begin{array}{l}\text { Family in which all spaces are } \\
\text { (H-all spaces) }\end{array}$ & 2B-2 & $\begin{array}{l}\text { A complex type of plan type 2A-2 in which } \\
\text { the entrance, living room, and hall are } \\
\text { inter-accessible (E-H, E-L, H-L) }\end{array}$ \\
\cline { 3 - 4 } & 2B-3 & $\begin{array}{l}\text { Addition of dressing room (R-P and MR-B are } \\
\text { changed to MR-P-B.) }\end{array}$ \\
\cline { 3 - 4 } & & $\begin{array}{l}\text { Living room, kitchen/dining room, and hall are } \\
\text { inter-connected, and the dressing room is located } \\
\text { beside the bathroom, as shown in plan type 2B-3 } \\
\text { (L-DK, L-H, DK-H, MR-P-B) }\end{array}$ \\
\hline
\end{tabular}

${ }^{*}$ Refers to a specific space. 

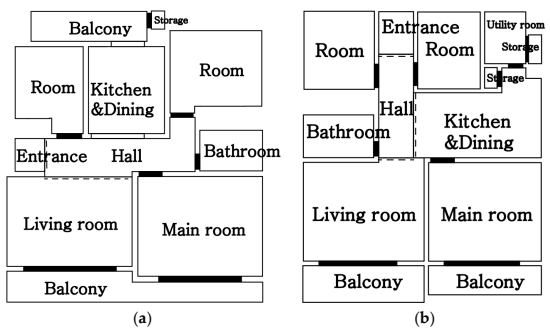

Figure 6. Ancestor plans of Family 2A and Family 2B. (a) Family 2A: Yeoksam Sungbo Apt. (1979); (b) Family 2B: Samick Green Apt. B type (1980).

In the next section, the features of each family are discussed.

\subsubsection{Family $2 \mathrm{~A}$}

Family 2A was branched into a plan type in which the entrance is only connected to the hall (2A-1, plan type 12,1988 ) and a plan type in which the entrance, hall, and living room were inter-connected (2A-2, plan type 10,1987), which was based on a prototype in which all spaces (rooms) were accessible through the kitchen/dining room (2A, plan type 1, 1979), as shown in Figure 7. Family 2A changes from plan type 1 (in which the main room was accessible through the kitchen/dining room) to plan type 3 (in which the bedrooms were accessible through the kitchen/dining room), which evolved into plan type 6 (in which the bathroom was standardized beside the main room).

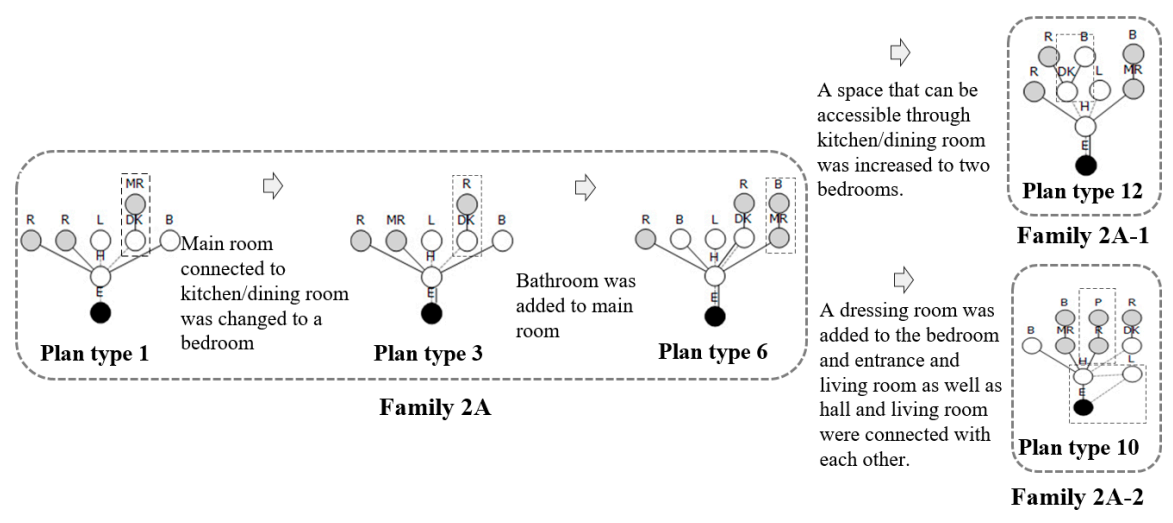

Figure 7. Transitions in spatial structures by Family 2A.

Family 2A-1 was characterized by a spatial structure in which the entrance was only connected to the hall (E-H). One difference in the spatial structure that changed between plan type 6 and plan type 12 was an increase in accessible spaces (two bedrooms) through the kitchen/dining room (DK-R, DK-R).

Family 2A-2 was a plan type in which the entrance, hall, and living room were inter-connected, as shown in plan type 10 in Figure 7. One difference in the spatial structure between plan type 6 and plan type 10 was the interconnection of the entrance, hall, and living room and the addition of a dressing room (R-P) to the bedroom connected to the hall.

\subsubsection{Family $2 B$}

Family 2B was developed from a spatial structure in which all spaces were accessible through the hall (plan type 2) and from plan type 4, in which the bathroom (MR-B) was standardized as shown in Figure 8. Family 2B-1 was characterized by a spatial structure, as shown in plan type 38, in which the number of bedrooms was temporarily reduced from three to two, and by plan type 45 , in which the bedrooms were accessible through the living room. Family 2B-2 combined 2B and 2A-2, in which all spaces were accessible through the hall (a characteristic of plan type $2 \mathrm{~B}$ ) and in which the entrance, 
living room, and hall were inter-accessible (a characteristic of plan type 2A-2). Family 2B-3 was characterized by a change in the dressing room location. The dressing room-which was connected to the general bedroom in plan type 26 (R-P) -was moved to a place between the main room and the bathroom (MR-P-B) in plan type 53. Family 2B-4 was characterized by inter-accessibility among the living room, kitchen/dining room, hall, and dressing room, which was beside the bathroom, as shown in 2B-3.

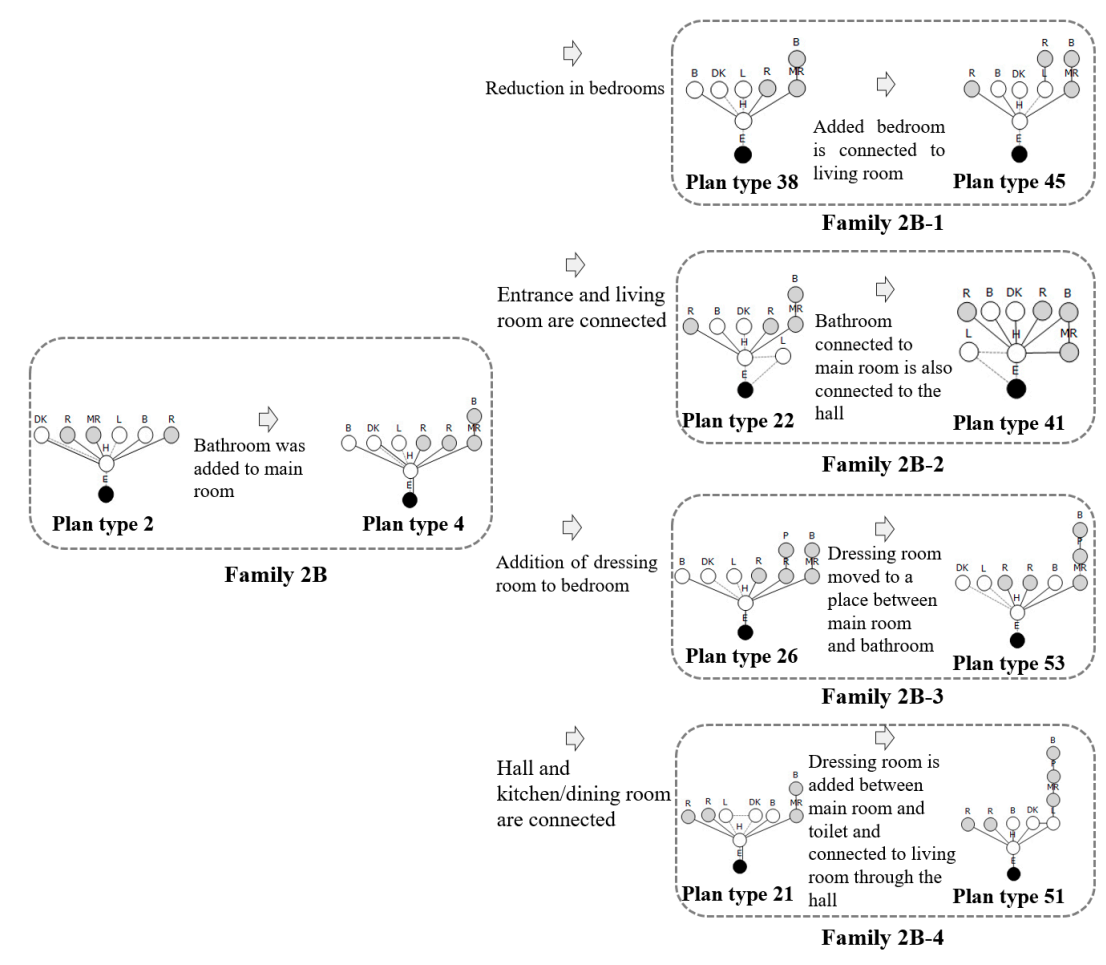

Figure 8. Transitions in spatial structures by Family 2B.

\subsection{Differentiation Analyses by Combinations of Rooms}

The meanings behind the changes in spatial organization by the families analyzed earlier are examined below. As mentioned earlier, changes in spatial organization are the result of diverse social and cultural factors. In the present study, only the factors that stem from differences and changes in spatial organization were addressed; other factors-such as the areas and locations of the spaces-were excluded. In order to analyze this meaning, we have extracted the genotypes defined by Bafna [11,12] in the premise of the study - that is, the combinations of rooms that lead to differences in the family. These combinations of rooms were classified, and the changes in number and ratio of each combination per year were tracked as shown in Figure 9 and Table A1 in the Appendix A. Based on these results, we explain the meaning of each combination.

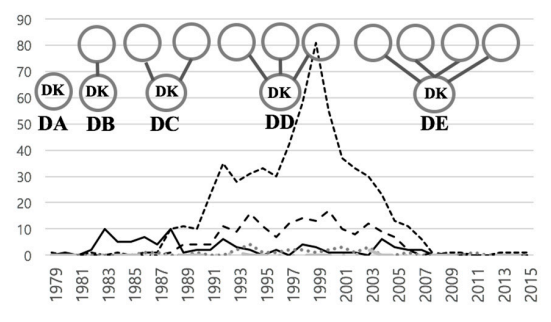

(a)

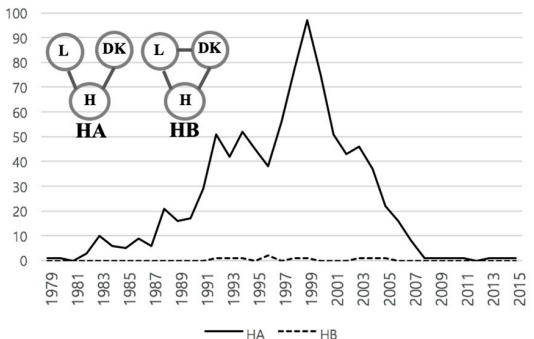

(b)

Figure 9. Cont. 


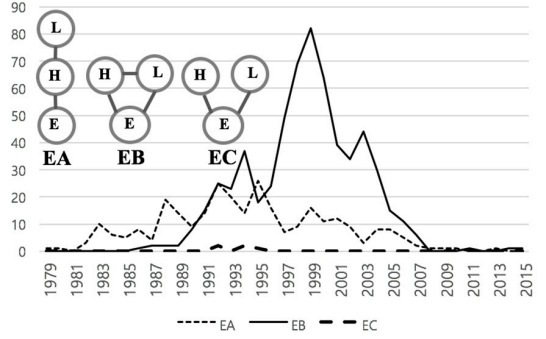

(c)

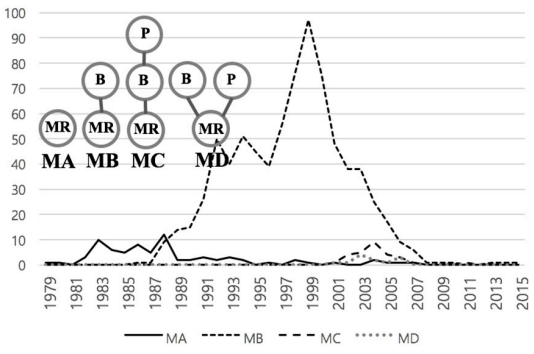

(d)

Figure 9. Transitions in spatial structures by combination of rooms. (a) The combinations of kitchen/dining room and other rooms; (b) The combinations of hall, living room, and kitchen/dining room; (c) The combinations of entrance, hall, and living room; (d) The combinations of main room, bathroom, and dressing room.

\subsubsection{An Area of Hall, Living Room, and Entrance}

In the examined area (as shown in Figure 3), rather than being a separate room, the hall is a space that exists between the living room and the kitchen, and provides a path to access individual rooms. Although the boundary between the living room and the kitchen is not clear, virtual compartments exist in the path that are secure. Therefore, although the hall is sometimes considered a part of the living room or the kitchen instead of a separate space, depending on the intention of the study, it is generally seen to have stemmed from the narrow wooden porches that used to run along the outside of rooms. In traditional architecture, narrow wooden porches running along the outside of rooms created a path that connected all the spaces together, even in apartments.

A common phenomenon in all types is the decrease in access through the hole, and the increase in access through the kitchen or living room, as shown in Figure 9a. This indicates that plans have been gradually changed from uniformly borrowing the idea of the narrow wooden porches that traditionally ran along the outside of rooms to facilitate direct access to commonly used spaces, such as the kitchen/dining room and the living room. Consequently, the rank of the hall has also gradually changed.

In this study, the relationships among entrance, hall, and living room were categorized as either adjacent (as shown in Figure 6a) or separate (as shown in Figure 6b). The decrease in type A and the increase in type B in Figure $9 \mathrm{~b}$ show a change in the entry direction of the room. By making this change, the lighting of the hall becomes better.

\subsubsection{An Area of Kitchen/Dining Room}

The kitchen, traditionally, is often a separate and independent space for cooking. Meals were eaten in the main room or in the living room. As houses began to be replaced by apartments, the spaces of the main room and the living room were differentiated, and a separate space for eating began to be included in the kitchen. Although some apartments are larger than the analyzed apartments-having an area of more than $115 \mathrm{~m}^{2}$ and a separate kitchen and dining room-in general, apartments have a combined kitchen/dining room used for both cooking and dining.

In Family 2A, the bedroom is accessed through the kitchen/dining room, which does not exist in traditional architecture. This can be regarded to have been universalized from the plans for initial high-grade apartments, which had a room for a kitchen maid that disappeared in the 1980s.

Family 2A-1 shows the process by which the importance of the kitchen/dining room space increased. The number of rooms accessed through kitchen/dining room has increased progressively since the late 1980s, as shown in the combinations of kitchen/dining room and other rooms of Figure 9a. Although not large in number, cases in which the kitchen/dining room is connected to the main room were found (i.e., plan types $16,17,37,40$, and 46 ). In general, the living room used to be a space in which family members gathered to enjoy leisure. However, as family members' personal lives 
and spaces became more important, as the time for family members to gather and eat became more important, and as females' opinions became more important than males' opinions in terms of apartment purchases, the reinforcement of the function of the kitchen/dining room has been reflected in modern apartment plans.

\subsubsection{An Area of Main Room}

As explained earlier, initial South Korean apartment plans were affected by detached house plans, which succeeded the spatial arrangement of traditional houses. In general, the floor in a traditional house which played the role of a living room was open, and the main room played the role of a public space for family members to eat meals and for visitors to be entertained. The main room of initial apartments was planned to be adjacent to the living room, considering the functions of the public space. However, as these functions were divided between the living room and the kitchen/dining room, and as the privacy of individual family members became more important, the main room has gradually become more likely to be planned to be a bedroom for couples.

This is similar to the practice in the early 1980s, when a bathroom dedicated to the main room was often installed to reinforce the function of the main room as the couple's bedroom. Installing a bathroom in the main room became more common in the late 1980s, as shown in the combinations of main room, bathroom, and dressing room (Figure 9d). This shows that the bedroom function of the parents' bedroom had been strengthened. This was also affected by the desire to possess two bathrooms, thereby enhancing the convenience for all family members.

Another characteristic of the main room is the dressing room, which is usually located between the main room and the main room's bathroom. The number of cases of adding dressing rooms has increased (as seen by Figure 9d), and have been incorporated into the plans since the early 2000s.

In general, in the case of detached houses, storage functions were secured by planning attic spaces in the upper parts of individual rooms. However, in the case of apartments, these storage spaces could not be easily secured, but were a main requirement of residents. As the main room was differentiated from a public space into a couple's bedroom, the main room could be reduced in terms of its area. In addition, as the desire to have the main room look more modern, storage, makeup, and dressing spaces were all differentiated; therefore, dressing rooms were more frequently planned for apartment spaces.

\section{Conclusions}

In the present study, a phylogenetic tree of families, in which the common types of apartments are shown, was created using a TBJ method for the architectural plans in order to analyze the types of spatial structures and the transition history of apartments in South Korea. The present study analyzed relationships between changes in the spatial structures of two-bay staircase apartments between 1979 and 2015.

There were two plan types for two-bay staircase apartments: Family 2A (DK-*), in which the specific spaces (rooms) were connected through the kitchen/dining room (DK); and Family 2B, in which all spaces were connected through the hall. Based on these basic types, the families changed gradually.

The characteristics of the target plans are branched and grouped based on the hall and kitchen/dining room. Bedrooms were originally accessible through the hall, but this gradually changed into access through the living room and kitchen/dining room. Because of this transition, the number of spaces that had to be passed to access each bedroom from the entrance increased.

The initial functions of the main room as a bedroom and a commonly used space have been reduced, now functioning more often as a couple's bedroom. A bathroom and a dressing room are often added to reinforce the functions of a couple's bedroom. In addition, the analyzed apartments were found to be concentrated on certain families and specific plan types. Plan (DK-O) 2A-in which 
other rooms are accessed through the kitchen/dining room-accounted for $90.2 \%$ (803) of all the plans and $69.0 \%(40)$ of all the plan types.

Apartment design was more sensitive to market demand than any other type of building because the supply of apartments is targeted to non-specific customers. Thus, a new plan type can be used continuously in the selection process, or can disappear from the market. Despite the contribution, the present study analyzed only apartments with areas of $85 \mathrm{~m}^{2}$, which is a limitation. For future research, more spatial structures will be analyzed to identify the characteristics of diverse structures.

Author Contributions: Sungil Ham collected and analyzed the data necessary for the research and wrote the original text. Hyunsoo Lee set the direction of the subject of the research and verified the errors of the research analysis.

Conflicts of Interest: The authors declare no conflict of interest.

\section{Appendix A}

Table A1. Number of cases where combinations have occurred by year.

\begin{tabular}{|c|c|c|c|c|c|c|c|c|c|c|c|c|c|c|}
\hline \multirow[t]{2}{*}{ Year } & \multicolumn{5}{|c|}{$\begin{array}{l}\text { Kitchen/Dining Room } \\
\text { and Other Rooms }\end{array}$} & \multicolumn{2}{|c|}{$\begin{array}{l}\text { Hall, Living Room, and } \\
\text { Kitchen/Dining Room }\end{array}$} & \multicolumn{3}{|c|}{$\begin{array}{c}\text { Entrance, Hall, } \\
\text { and Living Room }\end{array}$} & \multicolumn{4}{|c|}{$\begin{array}{l}\text { Main Room, Bath Room, } \\
\text { and Dressing Room }\end{array}$} \\
\hline & $\mathbf{A}$ & B & $\mathrm{C}$ & D & $E$ & $\mathbf{A}$ & B & A & B & $\mathrm{C}$ & $\mathbf{A}$ & B & $C$ & D \\
\hline 1979 & 0 & 1 & 0 & 0 & 0 & 1 & 0 & 1 & 0 & 0 & 1 & 0 & 0 & 0 \\
\hline 1980 & 1 & 0 & 0 & 0 & 0 & 1 & 0 & 1 & 0 & 0 & 1 & 0 & 0 & 0 \\
\hline 1981 & 0 & 0 & 0 & 0 & 0 & 0 & 0 & 0 & 0 & 0 & 0 & 0 & 0 & 0 \\
\hline 1982 & 2 & 1 & 0 & 0 & 0 & 3 & 0 & 3 & 0 & 0 & 3 & 0 & 0 & 0 \\
\hline 1983 & 10 & 0 & 0 & 0 & 0 & 10 & 0 & 10 & 0 & 0 & 10 & 0 & 0 & 0 \\
\hline 1984 & 5 & 1 & 0 & 0 & 0 & 6 & 0 & 6 & 0 & 0 & 6 & 0 & 0 & 0 \\
\hline 1985 & 5 & 0 & 0 & 0 & 0 & 5 & 0 & 5 & 0 & 0 & 5 & 0 & 0 & 0 \\
\hline 1986 & 7 & 1 & 0 & 1 & 0 & 9 & 0 & 8 & 1 & 0 & 8 & 1 & 0 & 0 \\
\hline 1987 & 4 & 1 & 0 & 1 & 0 & 6 & 0 & 4 & 2 & 0 & 5 & 1 & 0 & 0 \\
\hline 1988 & 10 & 10 & 1 & 0 & 0 & 21 & 0 & 19 & 2 & 0 & 12 & 9 & 0 & 0 \\
\hline 1989 & 1 & 11 & 4 & 0 & 0 & 16 & 0 & 14 & 2 & 0 & 2 & 14 & 0 & 0 \\
\hline 1990 & 2 & 10 & 4 & 1 & 0 & 17 & 0 & 9 & 8 & 0 & 2 & 15 & 0 & 0 \\
\hline 1991 & 2 & 23 & 4 & 0 & 0 & 29 & 0 & 14 & 15 & 0 & 3 & 26 & 0 & 0 \\
\hline 1992 & 6 & 35 & 11 & 0 & 0 & 51 & 1 & 25 & 25 & 2 & 2 & 50 & 0 & 0 \\
\hline 1993 & 3 & 28 & 9 & 2 & 1 & 42 & 1 & 20 & 23 & 0 & 3 & 40 & 0 & 0 \\
\hline 1994 & 2 & 31 & 16 & 4 & 0 & 52 & 1 & 15 & 37 & 1 & 2 & 51 & 0 & 0 \\
\hline 1995 & 0 & 33 & 11 & 1 & 0 & 45 & 0 & 26 & 18 & 1 & 0 & 45 & 0 & 0 \\
\hline 1996 & 2 & 30 & 7 & 1 & 0 & 38 & 2 & 16 & 24 & 0 & 1 & 39 & 0 & 0 \\
\hline 1997 & 0 & 42 & 12 & 2 & 0 & 56 & 0 & 7 & 49 & 0 & 0 & 56 & 0 & 0 \\
\hline 1998 & 4 & 58 & 14 & 2 & 0 & 77 & 1 & 9 & 69 & 0 & 2 & 76 & 0 & 0 \\
\hline 1999 & 3 & 81 & 13 & 1 & 0 & 97 & 1 & 16 & 82 & 0 & 1 & 97 & 0 & 0 \\
\hline 2000 & 1 & 55 & 17 & 2 & 0 & 75 & 0 & 11 & 64 & 0 & 0 & 75 & 0 & 0 \\
\hline 2001 & 1 & 37 & 10 & 3 & 0 & 51 & 0 & 12 & 39 & 0 & 1 & 48 & 1 & 1 \\
\hline 2002 & 1 & 33 & 8 & 1 & 0 & 43 & 0 & 9 & 34 & 0 & 0 & 38 & 4 & 1 \\
\hline 2003 & 0 & 30 & 12 & 3 & 2 & 46 & 1 & 3 & 44 & 0 & 0 & 38 & 5 & 4 \\
\hline 2004 & 6 & 23 & 9 & 0 & 0 & 37 & 1 & 8 & 30 & 0 & 2 & 25 & 9 & 2 \\
\hline 2005 & 3 & 13 & 7 & 0 & 0 & 22 & 1 & 8 & 15 & 0 & 1 & 17 & 4 & 1 \\
\hline 2006 & 2 & 11 & 2 & 1 & 0 & 16 & 0 & 5 & 11 & 0 & 1 & 9 & 3 & 3 \\
\hline 2007 & 2 & 6 & 0 & 0 & 0 & 8 & 0 & 2 & 6 & 0 & 1 & 6 & 1 & 0 \\
\hline 2008 & 0 & 0 & 1 & 0 & 0 & 1 & 0 & 1 & 0 & 0 & 0 & 1 & 0 & 0 \\
\hline 2009 & 0 & 1 & 0 & 0 & 0 & 1 & 0 & 1 & 0 & 0 & 0 & 1 & 0 & 0 \\
\hline 2010 & 0 & 1 & 0 & 0 & 0 & 1 & 0 & 1 & 0 & 0 & 0 & 1 & 0 & 0 \\
\hline 2011 & 0 & 0 & 0 & 1 & 0 & 1 & 0 & 0 & 1 & 0 & 0 & 0 & 1 & 0 \\
\hline 2012 & 0 & 0 & 0 & 0 & 0 & 0 & 0 & 0 & 0 & 0 & 0 & 0 & 0 & 0 \\
\hline 2013 & 0 & 1 & 0 & 0 & 0 & 1 & 0 & 1 & 0 & 0 & 0 & 1 & 0 & 0 \\
\hline 2014 & 0 & 1 & 0 & 0 & 0 & 1 & 0 & 0 & 1 & 0 & 0 & 1 & 0 & 0 \\
\hline 2015 & 0 & 1 & 0 & 0 & 0 & 1 & 0 & 0 & 1 & 0 & 0 & 1 & 0 & 0 \\
\hline
\end{tabular}




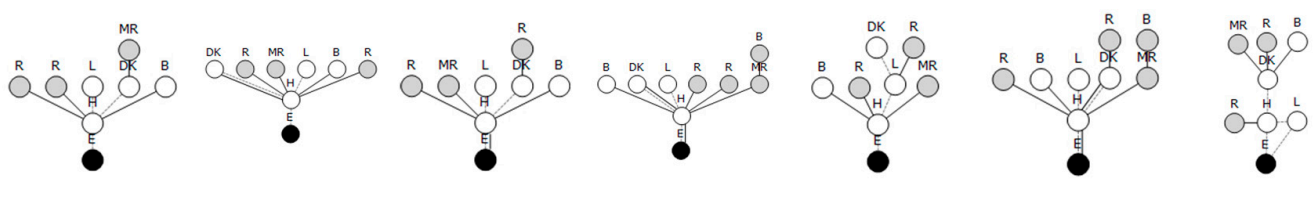

$\begin{array}{lllllll}1 & 2 & 3 & 4 & 5 & 6 & 7\end{array}$

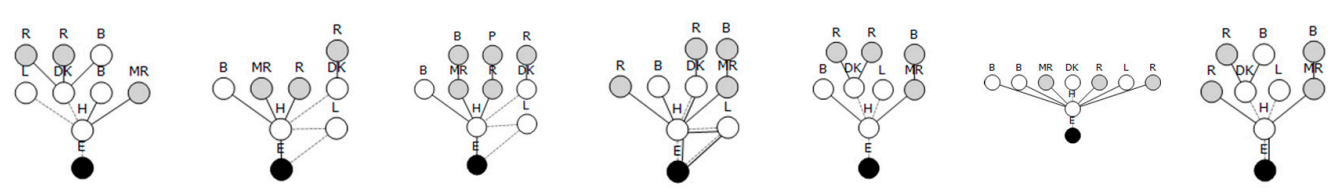

9

11

12

13

14
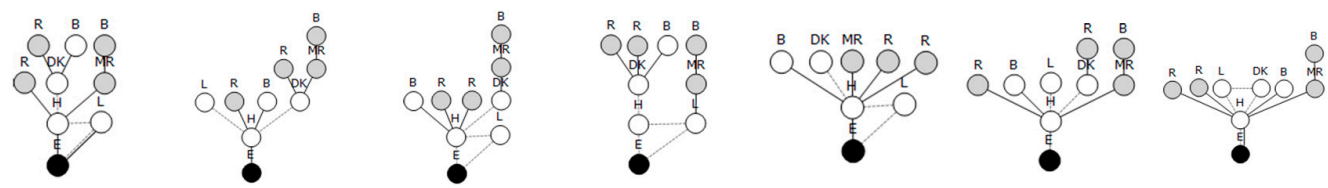

16

17

18

19

20

21
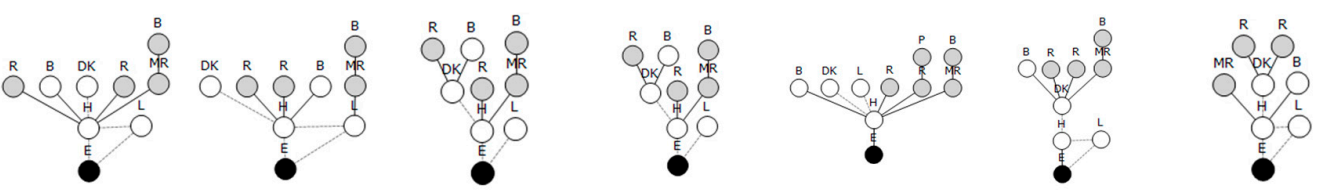

22

23

24

25

26

27

28

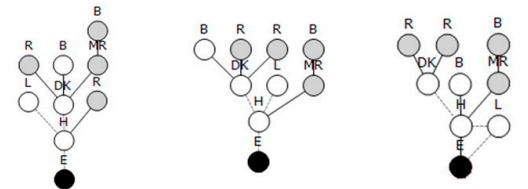

$29 \quad 30$

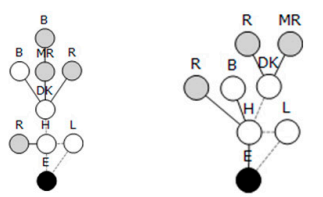

36

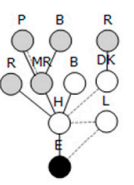

43

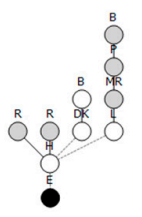

50

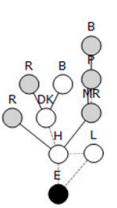

44

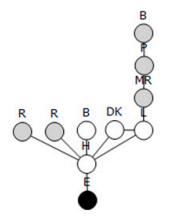

51

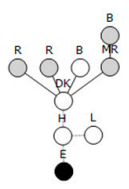

32

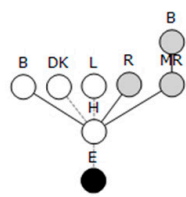

38

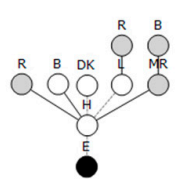

45

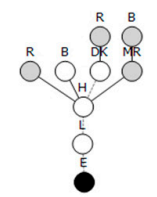

39

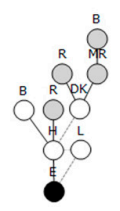

46

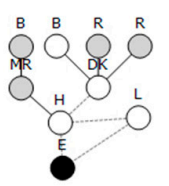

33

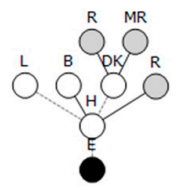

40

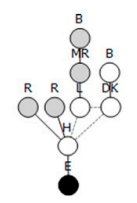

47

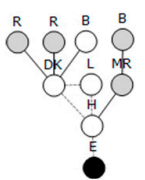

34

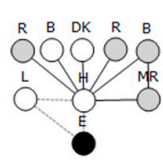

41

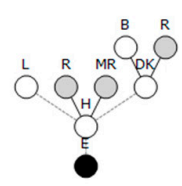

35

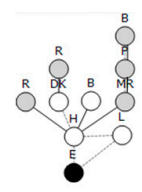

42

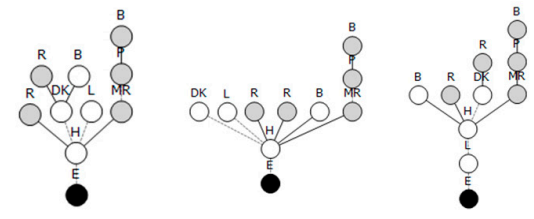

53

52

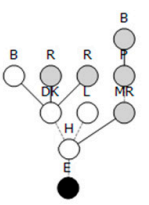

48

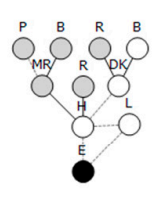

49

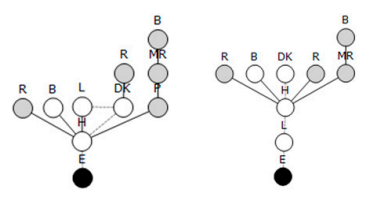

55

56

Figure A1. Spatial graph of plan types.

\section{References}

1. Statics Korea Population and Housing Census. Available online: http://kosis.kr/statHtml/statHtml.do? orgId=101\&tblId=DT_1JU0001\&conn_path=I2 (accessed on 30 July 2016). 
2. Lee, D. Spatial Configuration Characteristics of Apartment Units by Plan Size Using Genotype Analysis Method. Ph.D. Thesis, Yonsei University, Seoul, Korea, 2004.

3. Seo, K.W. Spatial Interpretation of Housing: The Role of Topological Intuition in the Evolution of the Houses in Seoul. Ph.D. Thesis, University College London, London, UK, 2005.

4. Bustard, W. Space, evolution, and function in the houses of Chaco Canyon. Environ. Plan. B Plan. Des. 1999, 26, 219-240. [CrossRef]

5. Bandyopadhyay, A.; Merchant, A.N. Space syntax analysis of colonial houses in India. Environ. Plan. B Plan. Des. 2006, 33, 923-942. [CrossRef]

6. Guney, Y.I.; Wineman, J. The evolving design of 20th-century apartments in Ankara. Environ. Plan. B Plan. Des. 2008, 35, 627-646. [CrossRef]

7. Hillier, B.; Hanson, J. The Social Logic of Space; Cambridge University Press: Cambridge, UK, 1984.

8. Ham, S.; Lee, G. Time-Based Joining Method for Generating Phylogenetic Trees of Architectural Plans. J. Comput. Civ. Eng. 2016. [CrossRef]

9. Ham, S. A Study on the Generation Method of a Phylogenetic Tree by the Similarity of Architectural Space. Ph.D. Thesis, Yonsei University, Seoul, Korea, 2011.

10. Ham, S. Analysis on the Change of Apartment House Spatial Structure Using Time Based-Joining Method-Focusing on Two-Unit-Staircase and 3-Bay Type Apartment House with an Area of Usable Space of $85 \mathrm{~m}^{2}$ Located in Seoul. J. Archit. Inst. Korea Plan. Des. 2016, 32, 3-10. [CrossRef]

11. Bafna, S. Geometrical intuitions of genotypes. In The 3rd Space Syntax, Symposium; College of Architecture, Georgia Institute of Technology: Atlanta, GA, USA, 2001; Available online: http://www.ucl.ac.uk/bartlett/ 3sss/papers_pdf/20_bafna.pdf (accessed on 27 December 2016).

12. Bafna, S. A Morphology of Intentions: An Interpretation of Mies Van Der Rohe's Residentia; College of Architecture, Georgia Institute of Technology: Atlanta, GA, USA, 2001.

13. Conroy Dalton, R.; Kirsan, C. Small-graph matching and building genotypes. Environ. Plan. B Plan. Des. 2008, 35, 810-830. [CrossRef]

14. Simpson, M.G. Plant Systematics; Elsevier Science: Burlington, MA, USA, 2010.

15. Sanfeliu, A.; Fu, K.-S. A distance measure between attributed relational graphs for pattern recognition. IEEE Trans. Syst. Man Cybern. 1983, 3, 353-362. [CrossRef]

16. Bunke, H. On a relation between graph edit distance and maximum common subgraph. Pattern Recogn. Lett. 1997, 18, 689-694. [CrossRef]

17. Kleinberg, J.M. Authoritative sources in a hyperlinked environment. J. ACM 1999, 46, 604-632. [CrossRef]

18. Melnik, S.; Garcia-Molina, H.; Rahm, E. Similarity flooding: A versatile graph matching algorithm and its application to schema matching. In Proceedings of the 18th International Conference on Data Engineering (ICDE), San Jose, CA, USA, 26 February-1 March 2002; pp. 117-128.

19. Jeh, G.; Widom, J. SimRank: A Measure of Structural-Context Similarity; Stanford InfoLab: Stanford, CA, USA, 2001.

20. Heymans, M.; Singh, A.K. Deriving phylogenetic trees from the similarity analysis of metabolic pathways. Bioinformatics 2003, 19 (Suppl. S1), i138-i146. [CrossRef] [PubMed]

21. Leicht, E.A.; Holme, P.; Newman, M.E.J. Vertex similarity in networks. Phys. Rev. E 2006, 73, 026120. [CrossRef] [PubMed]

22. Jeon, N.; Son, S.; Yang, S.; Hyeonguk, H. Social History of the Korea Housing/History of the Modern and Contemporary Korea Housing; Dolbege: Seoul, Korea, 2008; pp. 192-195.

23. Kang, B.; Kaing, I.; Park, G.; Park, I.; Park, C.; Baek, H.; Lee, G. History of the Korea Apartment Plan; Sejin: Seoul, Korea, 1999.

24. Kwon, H.-S.; Chun, W.-H.; Park, J.-Y. A study on the efficient utilization plan of balcony space in apartment. In the 2006 Fall Conference of the Architectural Institute of Korea; The Architectural Institute of Korea: Daegu, Korea, 2006; Volume 26, pp. 81-84. Available online: https://m.riss.kr/search/detail/DetailView.do?p_mat_ type $=1 \mathrm{a} 0202 \mathrm{e} 37 \mathrm{~d} 52 \mathrm{c} 72 \mathrm{~d} \&$ control_no=3639e7b58250d24cffe0bdc3ef48d419 (accessed on 27 December 2016). (In Korean)

(C) 2016 by the authors; licensee MDPI, Basel, Switzerland. This article is an open access article distributed under the terms and conditions of the Creative Commons Attribution (CC-BY) license (http:/ / creativecommons.org/licenses/by/4.0/). 